\title{
Thermodynamic and Transport Properties of Molten Bi-In Alloys
}

\author{
B. P. Singh ${ }^{1}$, B. K. Singh ${ }^{1}$, I. S. Jha ${ }^{3}$, G. K. Shrestha ${ }^{1}{ }^{4}$, I. Koirala ${ }^{1},^{2^{*}}$ \\ 1 University Department of Physics, T.M. Bhagalpur University, Bhagalpur, India \\ 2Central Department of Physics, Tribhuvan University, Kirtipur, Nepal \\ 3M.M.A.M. Campus, Biratnagar, Tribhuvan University, Nepal \\ 4Pulchok Engineering college, Pulchok, ,Lalitpur,Nepal \\ *Corresponding author: E-mail : ikphysicstu@gmail.com
}

\begin{abstract}
We have used simple statistical theory to describe the mixing behavior of liquid Bi-In alloys in terms of energetic and structure through the study of their thermodynamic and transport properties. The structural characteristics of Bi-In melts are described by the two microscopic functions, i.e. the concentration fluctuation in long wavelength limit and the Warren-Cowley short range order parameter. The transport properties are analyzed through the diffusion coefficient ratio and viscosity. The Gibb's free energy of mixing, enthalpy of mixing and entropy of mixing are the thermodynamic functions which are used to describe the thermodynamic behaviors. In whole analysis thermodynamic input parameter, i.e. interchange energy take important role which is temperature dependent. The computed results are in good agreement with experimental data and support a weak ordering tendency in molten Bi-In system.
\end{abstract}

Keywords: Simple statistical theory; Regular alloys; Microscopic functions; Transport properties

\section{INTRODUCTION}

The brittle nature of $\mathrm{Bi}$ as well as its tendency to segregation makes difficult the production of Bi-based alloys in the forms of wires or sheets. The Bi-In binary alloys are rarely used as solders, except a few particular compositions, while both, $\mathrm{Bi}$ and In, have great importance as a components of multi-component lead free solders containing $\mathrm{Ag}, \mathrm{Sn}, \mathrm{Sb}$ and $\mathrm{Zn}$, widely used in electronic industry [1-3]. Among these, alloys from the Bi-In-Sn ternary system have a wide range of fusible applications and are believed to be the most promising. The addition of $\mathrm{Sn}$ results in a significant improvement of mechanical properties [4]. It is also important to mention that Zn-based alloys containing the Bi-In inter-metallic compound are used to produce alkaline batteries, while Bi-In amalgams as a luminescent material improve handling characteristics of a fluorescent lamp [2, 5]. The energetics and the structure of the alloys melts as the way in which they are coupled are necessary prerequisites to understand the mixing behavior in liquid alloys. The nature of interactions and structural re-adjustment of the constituents in the liquid alloys can be deduced by combining the theoretical modeling of observable indicators such as the thermodynamic and thermophysical properties and the corresponding experimental data. Phase diagram has assured that Bi-In system exhibits the three intermetallic compounds: $\operatorname{BiIn}(\beta)$ with tetragonal structure, $\operatorname{Bi}_{3} \operatorname{In}_{5}(\gamma)$ with complex tetragonal structure and $\operatorname{BiIn}_{2}(\delta)$ with hexagonal structure [6-8]. Among the three phases, the Biln has the highest heat of formation and heat of fusion and it melts congruently at $383.15 \mathrm{~K}$ [8].

In the present work the thermodynamic and transport properties as well as the ordering phenomenon (the concentration fluctuation in long wavelength limit and the Warren-Cowley short range order parameter) of liquid BiIn alloys have been reproduced quantitatively using simple statistical theory in a weak interaction approximation. The grand partition function for the system has been solved under the frame work of such model to obtain analytical expressions for various parameters $[9,10]$. The theoretical formalism for the thermodynamic functions is presented in section 2.1, section 2.2 deals with structural function while the transport properties are presented in section 2.3. Result and discussions are given in section 3 . Section 4 outlines the conclusions.

\section{THEORETICAL FORMALISM}

\subsection{Thermodynamic functions \\ 2.1.1. Gibb's free energy of mixing, $G_{M}$}

The standard thermodynamic relation for Gibb's free energy of mixing $\left(\mathrm{G}_{\mathrm{M}}\right)$ is

$G_{M}=R T[x \ln x+(1-x) \ln (1-x)]+G_{M}^{X S}$ 
One can easily obtain a relation between excess free energy of mixing, $G_{M}^{X S}$ and the ratio of activity coefficients, $\gamma\left(=\gamma_{\mathrm{A}}\right.$ $\left./ \gamma_{\mathrm{B}}\right)$ by using standard thermodynamic relations [10].

$$
\begin{gathered}
\mathrm{G}_{\mathrm{M}}^{\mathrm{XS}}=\mathrm{RT} \int_{0}^{\mathrm{x}} \ln \gamma \mathrm{dx}=\mathrm{RT}\left[\mathrm{x} \ln \gamma_{\mathrm{A}}+(1-\mathrm{x}) \ln \gamma_{\mathrm{B}}\right] \\
\text { with } \gamma_{\mathrm{A}}=[(\beta-1+2 \mathrm{x}) / \mathrm{x}(1+\beta)]^{\mathrm{z} / 2} \\
\gamma_{\mathrm{B}}=[(\beta+1-2 \mathrm{x}) /(1-\mathrm{x})(1+\beta)]^{\mathrm{z} / 2}
\end{gathered}
$$

Where $\mathrm{R}$ is universal molar constant; $\mathrm{x}$, concentration of the component; $\omega$, interchange or ordering energy and $z$, coordination number.

Using the relations (1) and (2), one obtains an expression for the Gibb's free energy of mixing [8-11] as

$$
\begin{aligned}
\mathrm{G}_{\mathrm{M}} & =\mathrm{RT}\left[\mathrm{x} \ln \mathrm{x}+(1-\mathrm{x}) \ln (1-\mathrm{x})+\mathrm{x} \ln \gamma_{\mathrm{A}}+(1-\mathrm{x}) \ln \gamma_{\mathrm{B}}\right] \\
& =\mathrm{RT}\left[\mathrm{x} \ln \mathrm{x}+(1-\mathrm{x}) \ln (1-\mathrm{x})+\mathrm{x}(1-\mathrm{x}) \frac{\omega}{\mathrm{k}_{\mathrm{B}} \mathrm{T}}\right]
\end{aligned}
$$

\subsubsection{Enthalpy of mixing, $H_{M}$ and entropy of mixing, $S_{M}$}

The calculation of enthalpy and entropy of mixing are of immense relevance since they will likely to provide information on the temperature dependence of the ordering energy. The enthalpy of mixing $\left(\mathrm{H}_{\mathrm{M}}\right)$ can be obtained from the standard thermodynamic expression as

$$
\begin{aligned}
H_{M} & =G_{M}-T\left(\frac{\partial G_{M}}{\partial T}\right)_{x, P, N} \\
& =R T\left[x(1-x) \frac{\omega}{k_{B} T}-x(1-x) \frac{1}{k_{B}} \frac{d \omega}{d T}\right]
\end{aligned}
$$

And entropy of mixing $\left(\mathrm{S}_{\mathrm{M}}\right)$ can be worked out from the relation

$$
\mathrm{S}_{\mathrm{M}}=\left(\mathrm{H}_{\mathrm{M}}-\mathrm{G}_{\mathrm{M}}\right) / \mathrm{T}
$$

\subsection{Structural functions}

\subsubsection{Concentration-concentration fluctuations in the long wavelength limit, $S_{c c}(0)$}

$\mathrm{S}_{\mathrm{cc}}(0)$ is an essential structural function which has been widely used to study the nature of atomic order in binary liquid alloys[12]. It is thermodynamically related to Gibb's free energy of mixing $\left(\mathrm{G}_{\mathrm{M}}\right)$ through the equation

$S_{c c}(0)=R T\left(\frac{\partial^{2} G_{M}}{\partial x^{2}}\right)_{T, P, N}^{-1}$
Equations (1) and (6) gives the theoretical value of $\mathrm{S}_{\mathrm{cc}}(0)$ :

$S_{c c}(0)=x(1-x)[1+z(1-\beta) / 2 \beta]^{-1}$

For the ideal mixing, when the ordering energy is zero, concentration-concentration fluctuations are usually computed from:

$$
\mathrm{S}_{\mathrm{cc}}(0, \mathrm{id})=\mathrm{x}(1-\mathrm{x})
$$

\subsubsection{Warren-Cowley short range order parameter, $\alpha_{1}$}

The Warren-Cowley $[13,14]$ short-range order parameter $\left(\alpha_{1}\right)$ is another important structural function which is useful to quantify the degree of chemical order in the alloy at melt. It provides insight into the local arrangement of the atoms in the molten alloys. Although it is difficult to obtain the experimental values of $\alpha_{1}$, theoretical values of this parameter are easily obtain via conditional probability [A/B] which defines the probability of finding an A- atom as a nearest neighbor of a given B-atom. It can be evaluated theoretically $[13,14]$ :

$\alpha_{1}=(\mathrm{S}-1) /\{\mathrm{S}(\mathrm{z}-1)+1\}, \quad \mathrm{S}=\mathrm{S}_{\mathrm{cc}}(0) / \mathrm{S}_{\mathrm{cc}}^{\mathrm{id}}(0)$

where $\mathrm{z}$ is the coordination number, which is taken as 10 for our purposes.

\subsection{Transport properties}

The mixing behavior of binary liquid alloys can also be studied at the microscopic level in terms of transport properties such as viscosity, chemical diffusion coefficients etc.

\subsubsection{Viscosity}

Viscosity isotherm ( $\eta$ ) of liquid alloys is an important transport parameter for the understanding of the atomic level structure and interactions. As in the case of thermodynamic functions, the composition dependence of $\eta$ of liquid alloys is also found to exhibit either a linear variation, or positive or negative deviations from the linear law. A simple formula for the investigation of viscosity of a binary solution [15] is

$\eta=\eta_{\text {id }}\left[1-x(1-x)\left(\frac{2 \omega}{k_{B} T}\right)\right]$

with $\eta_{\text {id }}=x \eta_{\mathrm{A}}^{0}+(1-\mathrm{x}) \eta_{\mathrm{B}}^{0}$

where $\eta_{i}^{0}$ is the viscosity of pure component $i$ ( = A or B) and $\omega$ as usual, the interchange energy or order energy. 


\subsubsection{Diffusion}

$N K_{B} \operatorname{TLna}_{i}=\left(\frac{\partial G_{M}}{\partial N_{i}}\right)_{T . P . N}$

The mixing behavior of the alloys forming molten metal can also be studied at the microscopic level in terms of coefficient of diffusion. The mutual diffusion coefficient $\left(\mathrm{D}_{\mathrm{M}}\right)$ of binary liquid alloys can be expressed in terms of activity $\left(a_{i}\right)$ and self- diffusion coefficient $\left(\mathrm{D}_{\mathrm{id}}\right)$ of pure component with the help of Darken's equation [16]

$D_{M}=D_{i d} x_{i} \frac{d \ln a_{i}}{d x_{i}}$

We obtain an expression for $D_{M}$ in terms of $S_{c c}(0)$ [14]:

$\frac{D_{M}}{D_{\mathrm{d}}}=\frac{S_{\mathrm{c}}^{\mathrm{d}}(0)}{S_{\mathrm{c}}(0)}$

with $\quad D_{M}=x D_{B}+(1-x) D_{A}$

Where $\mathrm{D}_{\mathrm{A}}$ and $\mathrm{D}_{\mathrm{B}}$ are the self-diffusion coefficients of pure components A and B respectively. According to Eq. (14), $\mathrm{S}_{\mathrm{cc}}(0)<\mathrm{S}_{\mathrm{cc}}(0, \mathrm{id})$ is typical compound forming alloys implying $\mathrm{D}_{\mathrm{M}}>\mathrm{D}_{\text {id }}$. A peak on diffusivity curve, $\mathrm{D}_{\mathrm{M}} / \mathrm{D}_{\text {id }}$ verses $\mathrm{x}_{\mathrm{Bi}}$, suggests presence of maximum chemical order in the molten alloys system as well as the composition of the most probable associates formed in the liquid phase[14]. The relationship between $\mathrm{S}_{\mathrm{cc}}(0)$ and the diffusivity expressed by the ratio of the mutual and self-diffusion coefficients, $\mathrm{D}_{\mathrm{M}} / \mathrm{D}_{\mathrm{id}}$, indicates the mixing behavior of the alloys, i.e. the tendency for compound formation $\left(\mathrm{D}_{\mathrm{M}} / \mathrm{D}_{\mathrm{id}}>1\right)$ or phase separation $\left(\mathrm{D}_{\mathrm{M}} / \mathrm{D}_{\text {id }}<1\right)$ or ideal mixing $\left(\mathrm{D}_{\mathrm{M}} / \mathrm{D}_{\text {id }}\right.$ approaches 1). Considering only the energetics of the nearest- neighbor bond, equation (12) becomes

$$
D_{M}=D_{i d}\left[1-x(1-x)\left(\frac{2 \omega}{k_{B} T}\right)\right]
$$

\section{RESULT AND DISCUSSIONS}

In the present work, we have studied the composition dependence of the thermodynamic, structural and transport properties of Bi-In alloys at constant temperature $900 \mathrm{~K}$. It is understood from the theory that there are some parameters that need to be fitted in order to carry out the present calculations. The parameters are the coordination number $(z)$, interchange energy ( $\omega$ ) and temperature derivative of

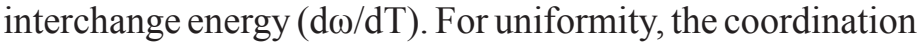
number $(z)$ in the liquid phase was chosen as 10 . We observed that the choice of $\mathrm{z}$ does not significantly affect our results. Interchange energy ( $\omega)$ and temperature derivative of interchange energy $(\mathrm{d} \omega / \mathrm{dT})$ for the alloys system have been estimated by the method of successive approximation using experimental data of free energy of mixing $\left(\mathrm{G}_{\mathrm{M}}\right)$ and enthalpy of mixing $\left(\mathrm{H}_{\mathrm{M}}\right)$ over the whole range of concentration[6]. Once suitable values of these parameters are chosen, the theory allows calculating other quantities mentioned above. The values of the fitted parameters for the alloys system are presented in Table 1.

\section{Table 1.}

Basic parameters

\begin{tabular}{ccccc}
\hline Alloys & $\mathrm{T}(\mathrm{K})$ & $\mathrm{z}$ & $\omega *$ & $\mathrm{~d} \omega / \mathrm{dT} *$ \\
\hline Bi-In & 900 & 10 & $-1.027 \times \mathrm{k}_{\mathrm{B}} \mathrm{T}$ & $-0.048 \times \mathrm{k}_{\mathrm{B}}$
\end{tabular}

*The values of these parameters are obtained by thermodynamic fitting.

\subsection{Thermodynamic properties}

Eqs.(3),(4) and (5) have been used to compute $\mathrm{G}_{\mathrm{M}} / \mathrm{RT}, \mathrm{H}_{\mathrm{M}} /$ $\mathrm{RT}$ and $\mathrm{S}_{\mathrm{M}} / \mathrm{R}$. Fig. 1 shows the result of these computations. We observed that there are remarkable agreement between computed and their respective experimental data [6]. The implication of $\omega$ in the Table 1 being negative for the BiIn system is that it is chemically ordered. Small values of $\omega$ suggest that they are weakly interacting. Fig.1 shows that there is remarkable agreement between computed $\mathrm{G}_{\mathrm{M}} /$ RT and their respective experimental data which confirms that the choice of $\omega$ for the alloys is quite reasonable. The plots (Fig.1) resulting from Eqs.(4) and (5) which incorporated temperature dependence of interchange energy $(\mathrm{d} \omega / \mathrm{dT})$ shows a remarkable agreement between the experimental data and the computed values which confirms the temperature dependence of the interchange energy of the alloys. The result of enthalpy of mixing $\mathrm{H}_{\mathrm{M}}$ /RT for Bi-In is shown in Fig.(1), compared with experimental results[6]. The experimental and computed values of $\mathrm{H}_{\mathrm{M}} / \mathrm{RT}$ show negative trend indicating clustering of unlike atoms or hetero- coordination. The results for the entropy of mixing for Bi-In are also plotted with concentration in Fig.(1). The positive value of $\mathrm{S}_{\mathrm{M}} / \mathrm{R}$ shows the presence of atomic order. 
More positive values of $S_{M} / R$ within entire concentration confirm that Bi-In system is hetero-coordinated alloys. The computed values of the entropy of mixing $\left(\mathrm{S}_{\mathrm{M}} / \mathrm{R}\right)$ are also compared with experimental results. We observed that there are also remarkable agreement between computed and their respective experimental data.

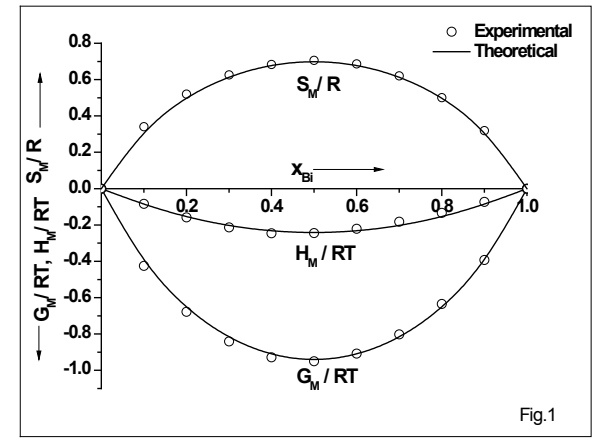

Figure 1-Free energy of mixing $\left(G_{M}\right)$, Heat of mixing $\left(H_{M}\right)$ and Entropy of mixing $\left(S_{M}\right)$ Vs Concentration of bismuth $\left(x_{B i}\right)$ for Bi-In liquid alloys at $900 \mathrm{~K}$. Solid line for theoretical values and circles for Experimental data[6].

\subsection{Structural properties}

We consider the calculated concentration fluctuation and Warren-Cowley chemical short range order parameter to understand the nature, strength and level of influence of the order in the properties of the alloys. The concentration fluctuation in long wavelength limit $\left(\mathrm{S}_{\mathrm{cc}}(0)\right)$ and the WarrenCowley chemical short range order parameter $\left(\alpha_{1}\right)$ have been computed via. Eqs.(7) and (9). We have used Eq.(7) to compute the $\mathrm{S}_{\text {cc }}(0)$ theoretically for the alloys. It can also be obtained directly from the measured activity [6] data as

$\mathrm{S}_{\mathrm{cc}}(0)=(1-\mathrm{x}) \mathrm{a}_{\mathrm{A}}\left(\frac{\partial \mathrm{a}_{\mathrm{A}}}{\partial \mathrm{x}}\right)^{-1}=\mathrm{xa}_{\mathrm{B}}\left(\frac{\partial \mathrm{a}_{\mathrm{B}}}{\partial(1-\mathrm{x})}\right)^{-1}$

where $a_{A}$ and $a_{B}$ are the observed chemical activities of constituents of the alloys. The $\mathrm{S}_{\text {cc }}(0)$, obtained from Eq.(16) are taken as experimental values. Figure 2 shows a plot of the computed and experimental values of $\mathrm{S}_{\mathrm{cc}}(0)$ along with the ideal values. The computed values of $\mathrm{S}_{\mathrm{cc}}(0)$ are in good agreement with the experimental values of $S_{c c}(0)$ for the alloys. The result can be used to understand the nature of atomic order in binary liquid alloys. At a given composition if $\mathrm{S}_{c c}(0)<\mathrm{S}_{\mathrm{cc}}(0$,id $)$ ordering in liquid alloy is expected while $S_{c c}(0)>S_{c c}(0, i d)$ gives the indication of tendency of segregation. $S_{c c}(0, i d)$ is the ideal value of $S_{c c}(0)$ which is associated with alloys of ideal mixture. In figure, it is seen that for Bi-In system $\mathrm{S}_{\mathrm{cc}}(0)<\mathrm{S}_{\mathrm{cc}}(0$,id $)$ which shows that liquid $\mathrm{Bi}-\mathrm{In}$ alloys at $900 \mathrm{~K}$ is of hetero-coordinating nature.

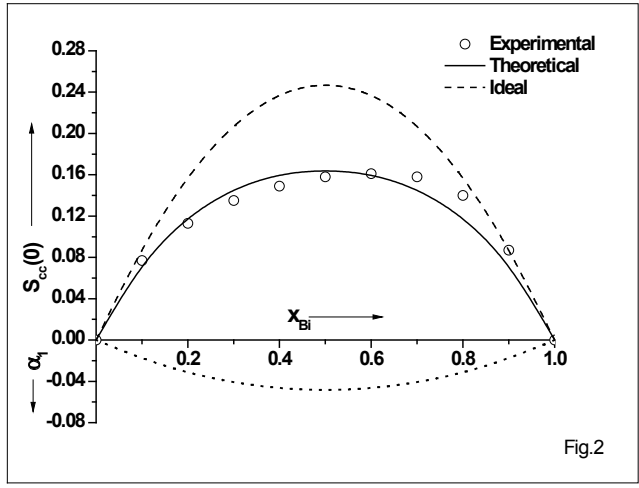

Figure 2-Concentration fluctuation at long wavelength limit $\left(S_{c c}(0)\right)$ and Warren-Cowley chemical short range order parameter $\left(\alpha_{1}\right)$ Vs Concentration of bismuth $\left(x_{B i}\right)$ for Bi-In liquid alloys at 900K. Solid line for theoretical values, circles for Experimental data [6] and dashed line for the ideal values of concentration fluctuation. Dotted line represents the Warren-Cowley chemical short range order parameter $\left(\alpha_{1}\right)$.

In addition, Warren-Cowley CSRO parameter $\left(\alpha_{1}\right)$ provides an immediate insight into the nature of the local arrangement of atoms in the mixture. The minimum possible value of $\alpha_{1}$ is -1 and it indicates complete ordering of unlike atom pairing at nearest atoms. On the other hand the maximum value of $\alpha_{1}$ is +1 , which implies complete segregation leading to phase separation and $\alpha_{1}=0$ corresponds to a random distribution of atoms. Figure 2 shows the plots of $\alpha_{1}$ against chemical composition, obtained from Eq.(9). In the Bi-In system, $\alpha_{1}$ is found negative throughout concentration of Bi.The negative values of $\alpha_{1}$ throughout whole concentration range of bismuth is the signatures of chemical ordering in the BiIn liquid alloys at $900 \mathrm{~K}$.

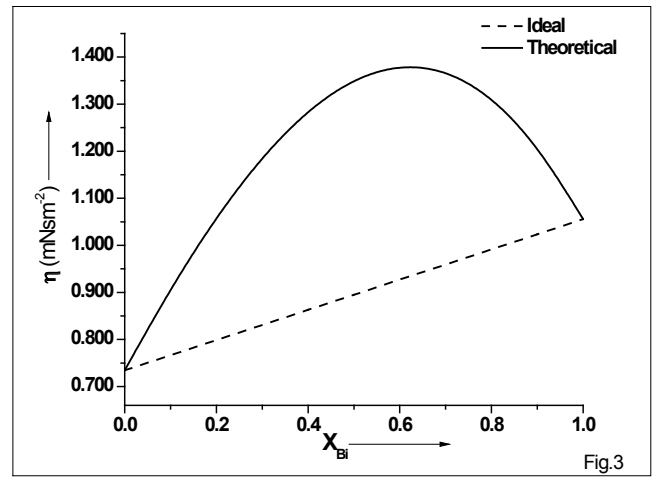

Figure 3-Viscosity $(\eta)$ Vs concentration of bismuth $\left(x_{B i}\right)$ in liquid Bi-In alloy at $900 \mathrm{~K}$; solid line for theoretical values and dotted line for ideal values. 


\subsection{Transport properties}

The viscosity of the Bi-In liquid alloy has been computed numerically from Eq. (10). From the plot of $\eta$ verses bulk concentration of $\mathrm{x}_{\mathrm{Bi}}$ (Fig.3) in Bi-In liquid alloy, positive deviation from the linear law (Raoult's law) in viscosity isotherms $\eta(\mathrm{x})$ have been observed for the regular alloy.

The calculated values of $S_{c c}(0)$ are used in Eq. (13) to evaluate the ratio of the mutual and intrinsic-diffusion coefficients, $\mathrm{D}_{\mathrm{M}} / \mathrm{D}_{\text {id }}$. For the consistency of the estimated order energy parameter, $\omega$, we have also calculated $\mathrm{D}_{\mathrm{M}} /$ $\mathrm{D}_{\text {id }}$ using Eq.(15). Fig.4 shows plot of $\mathrm{D}_{\mathrm{M}} / \mathrm{D}_{\text {id }}$ against the concentration of bismuth. In that plot the value of $\mathrm{D}_{\mathrm{M}} / \mathrm{D}_{\text {id }}$ is found greater than 1 in the entire range of concentration which is indicative for the compound formation in the mixture. A maximum value of $\mathrm{D}_{\mathrm{M}} / \mathrm{D}_{\text {id }}=1.56$ for $\mathrm{x}_{\mathrm{Bi}}=0.5$, confirms a weak tendency for chemical ordering, as observed by the $\mathrm{S}_{\mathrm{cc}}(0)$ and CSRO parameter.

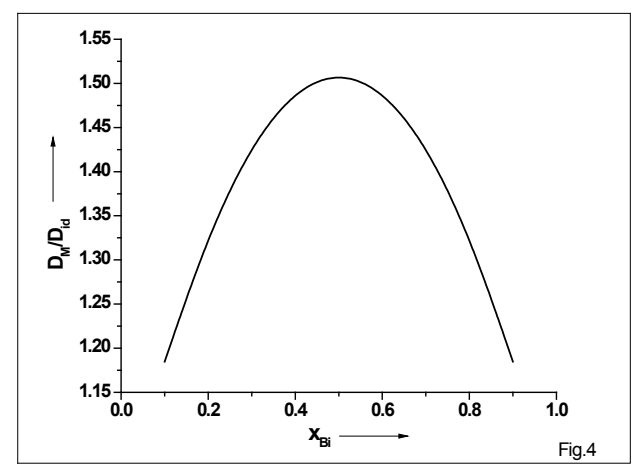

Figure 4- Ratio of chemical and intrinsic diffusion coefficients $\left(D_{M} / D_{i d}\right) V s$ concentration of bismuth $\left(x_{B i}\right)$ in liquid Bi-In alloy at $900 K$.

\section{CONCLUSIONS}

Following conclusions are drawn from the theoretical investigation on Bi-In liquid alloys at $900 \mathrm{~K}$ :

Order energy is small, negative and found to temperature dependent.

$>\quad$ The alloy system is weakly interacting and chemically ordered.

$>$ Thermodynamic and structural functions are deviated negatively from their ideality.

$>$ Viscosity isotherm of the alloy is found to deviate positively from linear law.

\section{REFERENCES}

[1] Kattner,U.R.,Handwerker,C.A.Z.Metallkde.2001, 92(7),740.

[2] Novakovic, R., Giuranno, D. , Ricci, E. , Lanata,T.,Amore S.CALPHAD. 2009,33, 69.

[3] Frear D.R.,Jang J.W.,Lin J.K.,Zhang, C. JOM.2001,53(6),28.

[4] Witusiewicz,V.T., Hecht,U., Bottger,B., Rex,S. J.Alloys Compounds.2007,428,115.

[5] Lankhorst, M.H.R., Keur, W., van Hal, H.A.M. J. Alloys Compounds. 2000, 309,188.

[6]Hultgren, R.,Desai, P.D.,Hawkins,D.T., Gleiser, M., Kelley, K.K. Selected Values of the Thermodynamic Properties of Binary Alloys, ASM Metals Park, Ohio. 1973.

[7] Massalski (Ed.) T.B., Binary Alloy Phase Diagrams, vol. 1, Am. Soc. for Metals, 1986, p. 510.

[8] Okamoto, H., Massalski, T.B. Phase Diagrams of Indium Alloys and Their Engineering Applications, in: C.E.T. White, H. Okamoto (Eds.), Monograph Series on Alloy Phase Diagrams, ASM International, Materials Park, Ohio, 1992, pp. 46-54.

[9] Singh, R.N., Mishra, I.K., Singh, V.N. Phys.Condens. Matter. 1990,2,8457.

[10] Koirala, I. , Singh, B.P. , Jha, I.S. J. Non-Cryst.Solids. 2014, 398-399,26-31.

[11] Koirala, I. , Jha, I.S., Singh, B.P. Bibechana. 2014, 11(1), 70.

[12] Bhatia, A.B. , Singh, R.N. Phys. Chem. Liq. 1982,11, 343.

[13] Warren, B.E. X-ray Diffraction, Addison-Wesle, Reading MA,1969.

[14] Cowley, J.M. Phys. Rev. 1950,77,667.

[15] Moelevyn-Hughes EA. Physical chemistry. Oxford, Pergamon; 1964.

[16] Darken LS, Gurry RW. Physical Chemistry of Metals. McGraw Hill, New York; 1953; 535. 